\title{
Complex Impedance Investigation of Epitaxial LCMO Thin Films
}

\author{
E. Mateev*, T. Nurgaliev and B. Blagoev \\ Institute of Electronics, Bulgarian Academy of Sciences, 72 Tzarigradsko Chaussee Blvd., 1784 Sofia, Bulgaria \\ (Received February 27, 2013; in final form May 15, 2014) \\ Epitaxial $\mathrm{La}_{0.67} \mathrm{Ca} .{ }_{33} \mathrm{MnO}_{3}$ (LCMO) thin films by off-axis magnetron sputtering were deposited on $\mathrm{SrTiO}_{3}$ \\ (STO) substrates. Complex impedance measurements (module and phase of the thin film impedance) in frequency \\ range $1-30 \mathrm{kHz}$ are done. Substantial dependence of both: module and phase of the complex surface impedance \\ is observed. The temperature interval scanned is from $77 \mathrm{~K}$ to room temperature. The impedance has inductive \\ behavior for temperatures less than the Curie temperature and capacitive for higher temperatures. Reduction of \\ the film thickness from 60 to $30 \mathrm{~nm}$ shifts the impedance curves to the lower temperatures.
}

DOI: $10.12693 /$ APhysPolA.126.787

PACS: 72.20.-i, 72.80.Ga, 72.90.+y, 73.61.Le

\section{Introduction}

The perovskite manganites are an object of experimental and theoretical studies due to several reasons [1]. Colossal magnetoresistance applications [2, 3], bolometric purposes, applications as a cathode material in solidoxide fuel cells [4].

Typical usage of the perovskite magnitoresistive materials is based on DC measurements. However, the same measurements can be done in the ac (above $1 \mathrm{kHz}$ ) range. The ac measurements can be more efficient, because the $1 / f$ noise is strongly reduced. This makes the investigation of the ac complex resistance interesting for the applications. In the same time they give a valuable knowledge on the individual contribution of grains and grain boundaries to the low field magnetoresistance $[5,6]$.

In [7] are presented the results of the complex impedance frequency dependence investigation in ac range (1-100 kHz) for mono- and polycrystal LCMO layers at room temperature (under the Curie temperature). Here we present the results of the complex impedance frequency dependence investigation in the region $1-30 \mathrm{kHz}$ for LCMO monocrystal layers in a temperature interval from $77 \mathrm{~K}$ to room temperature (the Curie temperature for LCMO is within this interval).

\section{Experimental}

The investigated layers were deposited on $5 \times 10 \mathrm{~mm}^{2}$ STO substrates by HF magnetron sputtering. The deposition temperature was $780^{\circ} \mathrm{C}$ and an oxidation step in $900 \mathrm{hPa} \mathrm{O}_{2}$ at $500^{\circ} \mathrm{C}$ succeeded the deposition. In $\mathrm{X}$-ray photoelectron spectroscopy (XPS) investigation of a film, deposited with the same technology is presented. It shows a good crystal quality of the film.

* corresponding author; e-mail: emateev@abv.bg
The scheme of the four point measurement of the thin film's complex impedance is shown in Fig. 1. An ac voltage generator is converted to ac current generator by means of a $1 \mathrm{M} \Omega$ resistor $R$. The current is approximately $U_{0} / R$, where $U_{0}$ is the ac voltage generated by the generator. The complex thin film's impedance of the layer is equal to $k R U / U_{0}$, is the complex value of the voltage drop between the 2nd and 3rd point in the 4 point set, measured by lock-in amplifier operating in differential mode. The form-factor $k$ depends on the geometry of the 4 point set and in our case $k \approx 3$.

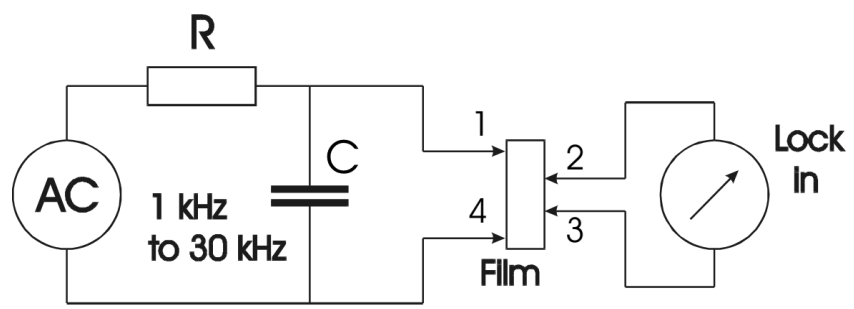

Fig. 1. Scheme of the four point measurement of the film's complex surface impedance.

In reality the situation is complicated by the fact that the resistance between the 1 st and 4 th points in the 4 point set can exceed $100 \mathrm{k} \Omega$ in the vicinity of the Curie temperature and reduce the current below $U_{0} / R$. Another factor to be taken into account is the parasitic capacitance of the wiring $C$. As a result it can be calculated that the current $i$, flowing through the film from point 1 to the point 2 of the four point structure is

$$
i=\frac{U_{0}-(1+\mathrm{j} \omega R C) k U}{R+r+\mathrm{j} \omega R r C},
$$

where $U_{0}$ is the ac generator's voltage, $U$ is the voltage measured between the points 2 and $3, r$ is the sum of the contact resistances at points 1 and $4, k$ is a formfactor (ratio between the surface resistance points $1-4$ to points $2-3)-$ in our case $k \approx 3$. The complex 
surface impedance is $U / I$ (our formfactor is $\approx 1$ ), and neglecting $r$ compared to $R$ we have

$$
\rho=\frac{U R}{U_{0}-k U-\mathrm{j} \omega R C k U} .
$$

The correction procedure has been verified by measuring two dummy objects at room temperature, each made from three equal resistors.

Two different thicknesses of the film were investigated: 30 and $60 \mathrm{~nm}$. The measurements were conducted in a temperature interval from $77 \mathrm{~K}$ to $300 \mathrm{~K}$, and frequencies from $1 \mathrm{kHz}$ to $30 \mathrm{kHz}$.

\section{Results and discussion}

The temperature dependence of the complex thin film impedance of $30 \mathrm{~nm}$ LCMO layer, deposited on STO substrate, for 5 different frequencies is shown in Fig. 2a and b. The modules are shown in Fig. 2a and the phases - in Fig. 2b. The modules show a maximum, corresponding to the approximately Curie temperature. The Curie temperature of the $30 \mathrm{~nm}$ epitaxial layer appears substantially less than the Curie temperature of the bulk material.
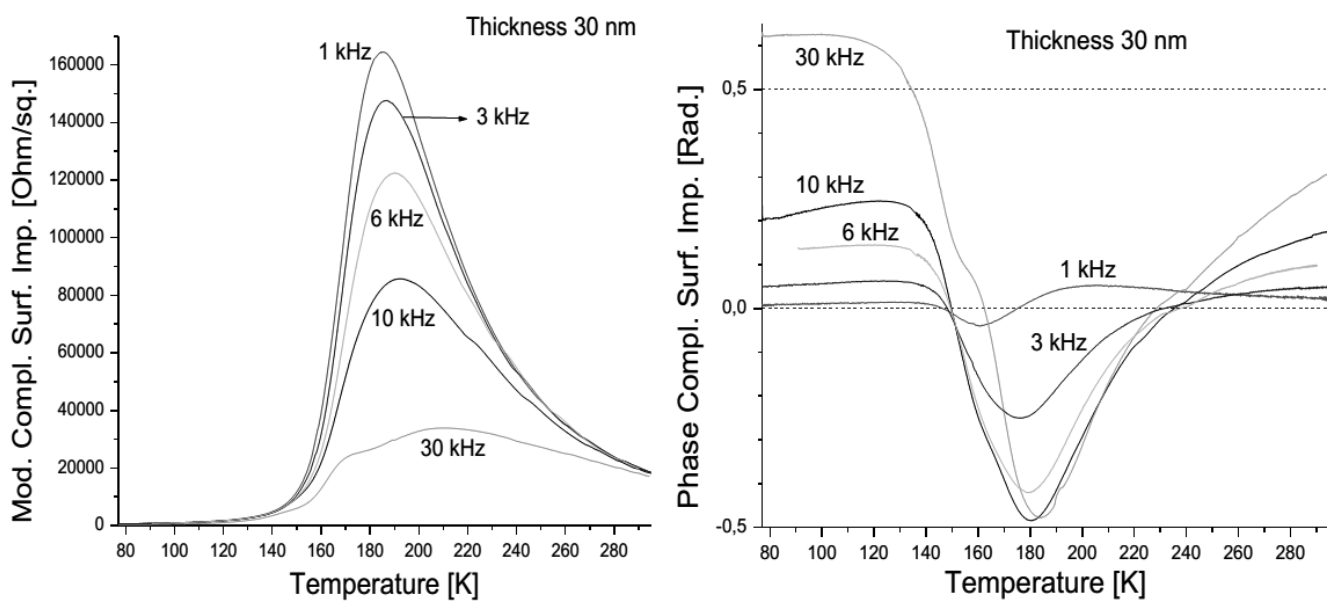

Fig. 2. (a, left) Modules of the thin film's impedance, (b, right) phases of the thin film's impedance.

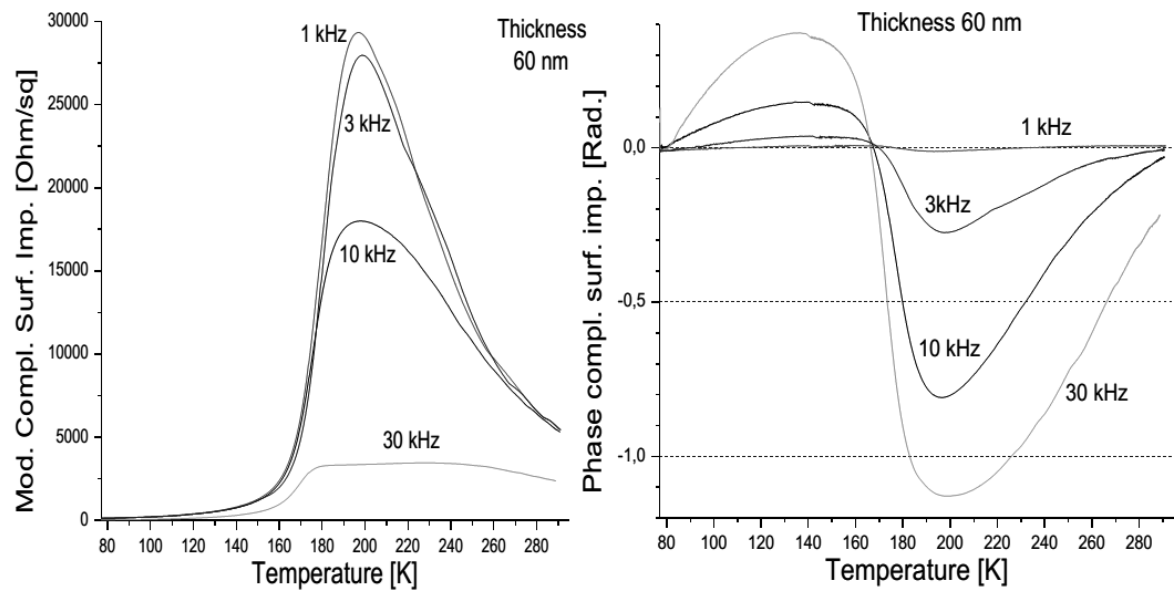

Fig. 3. (a, left) Modules of the thin film's impedance, (b, right) phases of the thin film's impedance.

The exact values of temperature of the peaks and their values (in $\mathrm{k} \Omega / \mathrm{sq}$ ) are shown in Table. The value of the impedance decreases with the frequency. The phase of the complex impedance is positive (inductive type) for low temperatures. This corresponds to our previously reported results of similar measurements with LCMO surface impedance measurements at room temperature - below the Curie temperature for LCMO [7]. It goes through zero at some temperature, becomes negative (capacitive type), and has a maximum at some temperature, higher than the temperature of the module maximum. The results of a measurement at $300 \mathrm{~Hz}$ are not shown: 
TABLE

The exact positions of the characteristic points of the curves, shown in Fig. 2a, 2b, 3a, 3b.

\begin{tabular}{|c|c|c|c|c|c|}
\hline & $1 \mathrm{kHz}$ & $3 \mathrm{kHz}$ & $6 \mathrm{kHz}$ & $10 \mathrm{kHz}$ & $30 \mathrm{kHz}$ \\
\hline max. module & 164.5 & 147.6 & 122.5 & 85.7 & 33.9 \\
\hline temp. $[\mathrm{K}]$ & 185 & 186.5 & 190 & 194 & 211 \\
\hline max. module & 29.4 & 27.95 & & 18 & 3.5 \\
\hline $\begin{array}{l}\text { thick. } 60 \mathrm{~nm} \\
\text { temp. }[\mathrm{K}]\end{array}$ & 196.5 & 198 & & 197.5 & 227 \\
\hline $\begin{array}{c}\text { max. pos. phase } \\
\text { thick. } 30 \mathrm{~nm}\end{array}$ & 0.015 & 0.06 & 0.15 & 0.25 & 0.62 \\
\hline max. pos. phase & 0.008 & 0.038 & & 0.15 & 0.37 \\
\hline temp. $[\mathrm{K}]$ & 143 & 142 & & 140 & 136 \\
\hline $\begin{array}{l}\text { temp. of zero } \\
\text { phase }-30 \mathrm{~nm}\end{array}$ & 146 & 147.5 & 149.4 & 149.6 & 164.6 \\
\hline $\begin{array}{c}\text { temp. of zero } \\
\text { phase }-60 \mathrm{~nm}\end{array}$ & 177 & 170 & & 167.5 & 166 \\
\hline $\begin{array}{c}\text { max. neg. phase } \\
\text { thick. } 30 \mathrm{~nm}\end{array}$ & 0.04 & 0.25 & 0.42 & 0.48 & 0.47 \\
\hline temp. $[\mathrm{K}]$ & 161 & 176 & 179.5 & 180.5 & 184.5 \\
\hline $\begin{array}{c}\text { max. neg. phase } \\
\text { thick. } 60 \mathrm{~nm}\end{array}$ & 0.011 & 0.275 & & 0.804 & 1.13 \\
\hline temp. $[\mathrm{K}]$ & 194 & 197.5 & & 196.5 & 199 \\
\hline
\end{tabular}

the modules curve coincides with the curve for $1 \mathrm{kHz}$, and the phase is zero (within the error limit). The exact temperatures and complex impedance values for the characteristic points are shown in Table. The temperature of the maximum impedance module is higher than the temperatures of complex impedance values characteristic points.

A capacitive nature of the impedance, observed in experiments, partly can be caused by temperature dependent permittivity of the STO substrate, where the LCMO film has been grown up. The effective dielectric constant of STO increases from $\approx 300$ to $\approx 2500$ when temperature is reduced from $\approx 300 \mathrm{~K}$ to $\approx 70 \mathrm{~K}[9-11]$. For this reason it is possible a forming of capacitive connections (through high permittivity STO component) not only between the adjacent grains of LCMO layer, but between different macroareas of LCMO layer as well. This leads to a strong increase of the contribution of the capacitive component to the summary impedance at temperatures, where the resistivity of the LCMO layer and the permittivity of the STO substrate are great (especially, at the Curie temperature of the sample) (Fig. 2a and b).

The temperature dependence of the complex thin film's impedance of $60 \mathrm{~nm}$ LCMO layer is shown in Fig. 3a and $b$. The modules are shown in Fig. 3a and the phases - in Fig. 3b. The exact temperatures and complex impedance values for the characteristic points are shown in Table. The modules of impedance of the $30 \mathrm{~nm}$ layer is much more than twice larger than the impedance of the $60 \mathrm{~nm}$ layer. The phase shows differences, too. The thin layer has inductive impedance even at $77 \mathrm{~K}$, while the thick layer starts with zero and has well expressed positive impedance phase maximum at higher temperatures - see Table. All characteristic temperatures in impedance temperature dependences are higher for $60 \mathrm{~nm}$ layer. The maximum negative phase is twice higher for the $60 \mathrm{~nm}$ layer.

\section{Conclusion}

The complex impedance of the epitaxial LCMO layers shows substantial frequency dependence even for relatively low frequencies -1 to $30 \mathrm{kHz}$. This cannot be explained by the common models for FM conductivity, which are dc theories.

The modules of impedance of the $30 \mathrm{~nm}$ layer larger than the impedance of the $60 \mathrm{~nm}$ layer. The phase shows differences, too. The thin layer has inductive impedance even at $77 \mathrm{~K}$, while the thick layer starts with zero and has well expressed positive impedance phase maximum at higher temperatures. All characteristic temperatures in impedance temperature dependences are higher for the thicker layer. The maximum negative phase is twice higher for the $60 \mathrm{~nm}$ layer. All these features show that the structure of thin layers becomes different from the structure of the thick layers (bulk material). A possible explanation is that when the layer thickness becomes close to or less than the ferromagnetic domains' size their structure can change. It should be noted also that not only the structural characteristics of the substrates, but their physical (electrical) properties can affect the electrical characteristics of the films grown on them.

\section{References}

[1] V. Strbik, M. Spankova, M. Reifers, J. Kovac, S. Benacka, J. Phys., Conf. Series 223, 012027 (2010).

[2] E.L. Nagaev, Usp. Phys. Nauk 166, 92 (1996).

[3] S.Y. Yang, W.L. Kuang, Y. Liou, W.S. Tse, S.F. Lee, Y.D. Yao, J. Magn. Magn. Mater. 268, 326 (2004).

[4] H. Rahmouni, M. Nouiri, R. Jemai, N. Kallel, F. Rzigua, J. Magn. Magn. Mater. 316, 23 (2007).

[5] I.-B. Shim, K.-T. Park, C.-S. Kim, Y.-J. Oh, J. Magn. Magn. Mater. 226-230, 733 (2001).

[6] H.M. Lee, I.-B. Shim, C.S. Kim, J. Magn. Magn. Mater. 282, 291 (2004).

[7] E. Mateev, B. Blagoev, T. Nurgaliev, J. Phys. Conf. Ser. 356, 012022 (2012).

[8] B.S. Blagoev, I.G. Gostev, T.K. Nurgaliev, V. Strbik, I.E. Bineva, L. Uspenskaya, E.S. Mateev, L. Neshkov, E. Dobročka, Š. Chromik, Conf. Series 514, 012041 (2014).

[9] H.E. Weaver, J. Phys. Chem. Solids 11, 274 (1959).

[10] Ch. Ang, Z. Yu, L.E. Cross, R. Guo, A.S. Bhalla, Appl. Phys. Lett. 79, 818 (2001).

[11] D. Fuchs, C.W. Schneider, R. Schneider, H. Rietschel, J. Appl. Phys. 85, 7362 (1999). 